\title{
Happy New Year
}

On the threshold of the New Year we think back to the past 12 months and provide a few highlights.

$\Lambda$ $t$ the opening of our fourth volume, as has become our tradition at Nature Catalysis, we are taking the opportunity to reflect on the complex yet exciting year that has just concluded.

It goes without saying that 2020 was dominated by the COVID-19 emergency. The disease, which was characterized as a pandemic by the WHO on 11th March, has profoundly affected our lives with a number of serious social and economic ramifications ${ }^{1}$. And while we now look to the rollout of the first vaccine campaigns ${ }^{2}$ as the light at the end of the tunnel, patience and caution must still accompany us in the coming months.

Despite the difficulties, however, the catalysis community at large has managed to thrive and we have had the pleasure of considering and publishing a remarkable selection of top-quality articles from across the entire field. A huge thank you goes to all our authors, reviewers and readers for their dedication under such difficult circumstances.

\section{"Despite the difficulties, however, the catalysis community at large has managed to thrive and we have had the pleasure of considering and publishing a remarkable selection of top-quality articles from across the entire field."}

The third volume of Nature Catalysis contains 104 articles, including primary research Articles, Review Articles and Perspectives. The distribution in terms of topics - broadly classified into five major subject areas (pictured) - remains consistent with the trends observed in previous years $\mathrm{s}^{3,4}$. Works in the area of homogeneous catalysis have acquired a good share of the overall publications; in contrast, manuscripts categorized as heterogeneous catalysis - mostly thermal catalysis with porous or supported catalysts - are slightly decreased in percentage. However, considering that $\sim 87 \%$ of all electrocatalysis

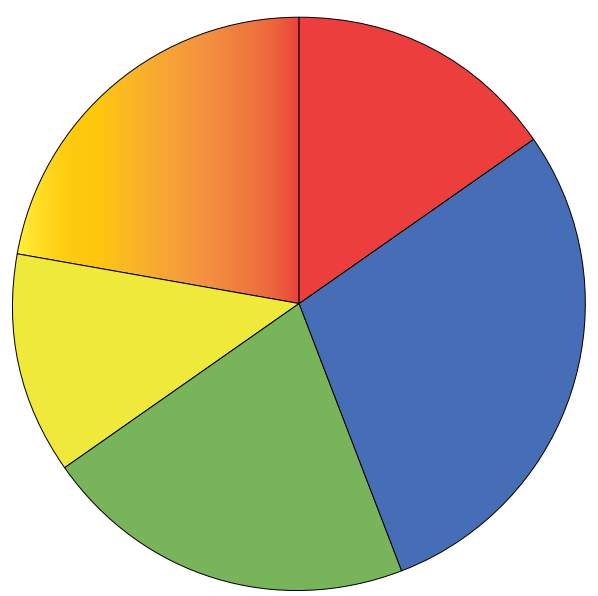

potential application to catalytic processes that may contribute to a more sustainable society. In keeping with our commitment to support the Sustainable Development Goals, the November issue featured two manuscripts and a Comment dedicated to plastic upcycling, which are highly related to the Responsible Production and Consumption goal.

One of these articles, which deals with the catalytic deconstruction of plastic waste into hydrogen and high-value carbons under microwave irradiation, belongs to the top two articles published in Nature Catalysis in 2020, according to Altmetric. This and a second article, which discusses the preparation of a spatially orthogonal hierarchically porous catalyst and its application in cascade and antagonistic reactions, have received Altmetric scores of above 200, an indication of the high level of attention received online; for example, in media, blogs and social networks.

While the past year has been full of challenges and successes, we are looking forward to exciting developments in catalysis in the coming months. But besides the science, some news is also coming on the publishing front. As of January, we have begun our journey towards transitioning to fully open access by operating as a Transformative Journal. Accordingly, authors will now have the option to publish their articles following the gold open access model in addition to the traditional publication route based on subscription. We hope these options will effectively enable authors to publish their work according to their preferred model, or to fulfil funder and institutional requirements where needed.

Finally, we hope you enjoy reading the articles selected for this issue and wish you a Happy New Year.

Published online: 21 January 2021

https://doi.org/10.1038/s41929-021-00573-6

References

1. Nat. Catal. 3, 331 (2020); https://doi.org/10.1038/s41929-0200458-5

2. Cohen, J. \& Normile, D. 'It's crazy.' Upbeat COVID-19 vaccine news from China and U. K. leaves scientists wanting more details. Science https://doi.org/10.1126/science.abg3694 (2020).

3. Nat. Catal. 2, 1 (2019); https://doi.org/10.1038/s41929-0190228-4

4. Nat. Catal. 3, 1 (2020); https://doi.org/10.1038/s41929-020$0424-2$ 